\title{
Magnetic alignment of nonmagnetic silicates caused by paramagnetic anisotropy: origin of polarization observed in planetary formation region
}

\author{
Chiaki Uyeda, Keiji Hisayoshi, and Shun Kanou \\ Department of Earth and Space Sciences, Graduate School of Sciences, Osaka University, \\ Machikaneyama 1-1, Toyonaka, Osaka 560-0042, Japan
}

(Received July 31, 2008; Revised November 30, 2008; Accepted December 2, 2008; Online published February 12, 2010)

\begin{abstract}
Possible dust alignment in dense regions due to paramagnetic anisotropy $\Delta \chi_{\text {PARA }}$ is discussed based on the results of a laboratory experiment on ortho-pyroxene grains containing a small amount of $\mathrm{Fe}^{2+}$ ions $(1.8 \mathrm{~mol} \%)$. Ortho-pyroxene has been detected in circum-stellar regions by infrared emission spectroscopy. Our experiment was performed at room temperature using $\mathrm{He}$ gas as the dispersing medium. Although the grains do not contain strong magnetic moments, alignment was achieved at low field strength $(<2000 \mathrm{G})$. The alignment efficiency of ortho-pyroxene was compared with those of various rock-forming minerals. The magnitude of diamagnetic anisotropy $\Delta \chi_{\text {DIA }}$, free of paramagnetic ions, is generally $<10^{-8} \mathrm{emu} / \mathrm{g}$ for various silicates. In contrast, $\Delta \chi_{\text {PARA }}$ increases considerably with increasing $\mathrm{Fe}^{2+}$ concentration, reaching $10^{-5} \mathrm{emu} / \mathrm{g}$ for many of the silicates when the concentration of $\mathrm{Fe}^{2+}$ is $>1 \mathrm{~mol} \%$; this increasing $\Delta \chi_{\text {PARA }}$ is the cause of the above-mentioned alignment obtained at the low field strength. Based on our observations, we infer the field intensity needed to obtain partial dust alignment of sufficient size to explain the observed polarization in astrophysical environments. Due to temperature dependences caused by a Curie Law and a rotational Brownian motion, the field intensity required to cause the alignment is expected to decrease considerably at low-temperature conditions assumed for a proto-planetary disk. The results of our experiment performed at room temperature provide a technical basis to reproduce grain alignment under such temperature conditions.
\end{abstract}

Key words: Magnetic field direction, dust alignment, ortho-pyroxene grains, proto-planetary disks, paramagnetic anisotropy.

\section{Introduction}

The direction of magnetic field $B$ in a diffuse region is frequently estimated from infrared or visible polarization $P$ caused by partial dust alignment; a weak galactic field of approx. $3 \mu \mathrm{G}$ is considered to be the cause of alignment. Because the geometrical relationship between $P$ and $B$ strongly depends on the mechanisms of alignment, it is important to firmly establish this mechanism to obtain a reliable field direction from polarimetry data. The conventional mechanism of alignment (the so-called a "DavisGreenstein model") is based on a paramagnetic relaxation process of magnetic moments included in the dust (e.g. Spizer, 1978). This model requires an extremely high rotational energy relative to the thermal energy - a condition that is only met in a diffuse interstellar region. The model is not realistic for planetary formation regions wherein thermal equilibrium is achieved between dust and gas (Whittet, 1992). Consequently, alternative mechanisms for the origin of magnetic alignment should be considered for solid materials that do not possess a strong magnetic moment. According to data compiled on solar elemental abundance and

Copyright (C) The Society of Geomagnetism and Earth, Planetary and Space Sciences (SGEPSS); The Seismological Society of Japan; The Volcanological Society of Japan; The Geodetic Society of Japan; The Japanese Society for Planetary Sciences; TERRAPUB.

doi:10.5047/eps.2008.12.003 infrared emission observational data, high concentrations of magnetic ions cannot be expected in the dust particles. The current theory of magnetic alignment of solid particles is that it occurs only for materials possessing a strong magnetic moment.

Alignment of micron-size crystals caused by the anisotropy of magnetic susceptibility $\Delta \chi$ was recently observed for common rock-forming silicates, namely kaolinite, talc, muscovite and biotite (Uyeda et al., 1991, 1993). The experiment was performed at room temperature $(T=$ $300 \mathrm{~K}$ ) using liquid ethanol as the dispersing medium. Partial alignment occurred by a simple balance between Brownian energy $1 / 2 k_{\mathrm{B}} T$ and field-induced anisotropy energy $1 / 2 M \Delta \chi B^{2}$, as was first proposed by Langevin and Curie (1910); where $M$ denotes the mass of the particle and $\Delta \chi$ describes anisotropy per unit mass. This process of grain alignment has been quantitatively studied on inorganic material, such as clay and mica minerals (Uyeda et al., 1993, 2003, 2005a) where it was explicitly shown that alignment was controlled by $\Delta \chi, T$ and $M$. This result means that the minimum field intensity required to achieve alignment is predictable, provided that the numerical values of these three parameters can be determined. This mechanism of aligning nonmagnetic particles was considered to be a promising technique to develop new types of industrial material (e.g. Maret and Dransfield, 1985). When an axis of 
a grain material possesses high material functionality, such as in terms of hardness, conductivity or optical efficiency, among others, this functionality can also be preserved in a grain aggregate provided that the grain axes are orientated in one direction. Based on this principle, attempts were made to produce large single crystals of protein, synthetic bone materials or aggregations of carbon nano-tubes. However, to date, only a limited number of materials have been investigated. Alignment is believed to occur only at a field intensity $>10,000 \mathrm{G}$, which requires a superconducting magnet.

Recent observations of infrared emissions have enabled the dust in the planetary formation region to be identified as rock-forming minerals, such as forsterite, ortho-pyroxene, diopside or hibonite; these are frequently observed in chondritic meteorites (e.g., Hanner et al., 1995; Malfait et al., 1998; Sitko et al., 1999; Honda et al., 2003; Hofmeister et al., 2003). The observations indicated that these silicates had Mg-rich components. Based on the observed $69-\mu \mathrm{m}$ feature, the $\mathrm{Fe}$ concentration of the forsterite grains was estimated to be $<5 \%$ (e.g. Molster et al., 2002). On the other hand, a small shift observed for the 11.44- $\mu \mathrm{m}$ feature of Vega-like star HD 14563 indicated the existence of Fe-bearing forsterite (Honda et al., 2004). Fe-bearing silicates are commonly seen in chondritic meteorites as well as in interplanetary dust particles (IDP) that are derived from the early solar system. It is likely that sufficient amount of silicate dusts in the circum-stellar region contain a small amount of $\mathrm{Fe}^{2+}$ ions (below several percent).

Here we report on an experimental study on the process of magnetic alignment. The experiment was carried out at room temperature on micron-sized ortho-pyroxene crystals dispersed in He gas, and the result was analyzed based on the $\Delta \chi$ values measured for bulk single crystals. Because temperatures of proto-planetary disks are estimated to range between $T=400 \mathrm{~K}$ and $50 \mathrm{~K}$ at a radius between $2 \mathrm{AU}$ and $50 \mathrm{AU}$ (Willacy and Langer, 2000), we discuss the effect of reducing the temperature on the magnetic alignment process. The practicability of dust alignment due to the above-mentioned process proposed by Langevin and Curie is quantitatively evaluated for the proto-planetary disks since reliable values of the three parameters are obtained for this region; values of $T$ and $M$ (dust size) are estimated from astronomical observation, whereas the $\Delta \chi$ value is determined by the magnetic-anisotropy measurement of a bulk single crystal composed of the same material as that identified in proto-planetary disks.

\section{Experimental}

A method to measure the alignment process in He gas recently developed by Uyeda et al. (2001) is shown schematically in Fig. 1. A large Dewar (inner diameter: 150 $\mathrm{mm}$; inner height: $400 \mathrm{~mm}$ ) serves as a sample chamber. This cell size was necessary to maintain the dispersed particles in the gas phase during the time of the experiment. The cell was placed at the center of a Helmholtz coil system having an inner diameter $1000 \mathrm{~mm}$; the coil produced a homogeneous field up to $700 \mathrm{G}$ within a spherical area $500 \mathrm{~mm}$ in diameter at the center of the system. The inhomogeneity of the field was less than $1000 \mathrm{ppm}$

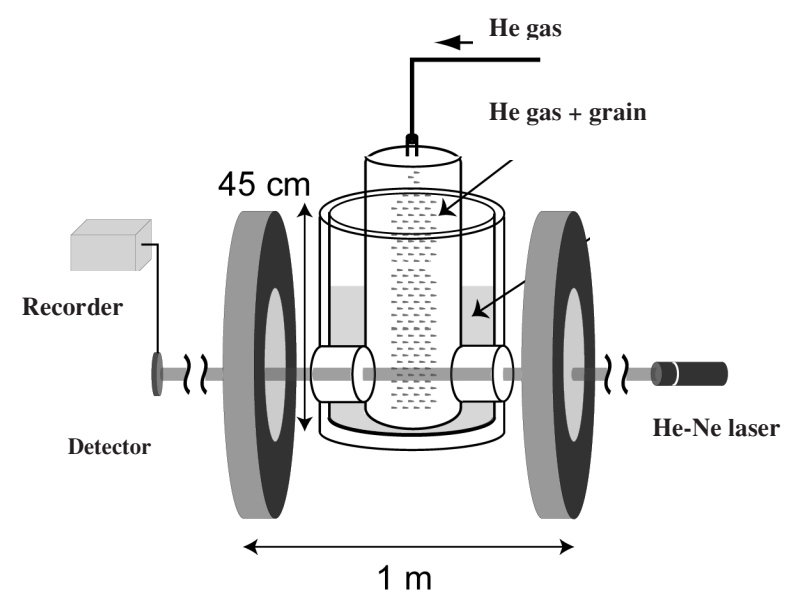

Fig. 1. Schematic view of apparatus developed to measure magnetic grain alignment under low-temperature conditions between $T=100 \mathrm{~K}$ and $10 \mathrm{~K}$ (Uyeda et al., 2001, 2004c). He gas is used as the dispersing medium. A set of Helmholz coils produces a homogeneous magnetic field in a spherical area of $100 \mathrm{~mm}$ in diameter; the sample suspension is included in this area. Alignment of graphite grain at $T=110 \mathrm{~K}$ was recently performed using this setup (Uyeda et al., 2004c).

inside the sphere. The interior of the Dewar was placed where the field is homogeneous. Single-crystal grains of ortho-enstatite $\left(\mathrm{Mg}_{0.983}, \mathrm{Fe}_{0.017}\right.$, from Morogoro, Tanzania) were used. The sample was free of ferromagnetic impurities and contained $1.8 \mathrm{~mol} \%$ paramagnetic $\mathrm{Fe}^{2+}$ ions. For the bulk single crystal, $\chi$ at $T=300 \mathrm{~K}$ was measured using standard procedures (Uyeda et al., 2005b). Measured anisotropy between the $c-a, c-b$ and $b-a$ axes were $\Delta \chi_{c-a}=1.2 \times 10^{-5} \mathrm{emu} / \mathrm{g}, \Delta \chi_{c-b}=1.0 \times 10^{-5} \mathrm{emu} / \mathrm{g}$ and $\Delta \chi_{b-a}=0.2 \times 10^{-5} \mathrm{emu} / \mathrm{g}$, respectively. Magnetic susceptibility of the sample measured by a vibrating sample magnetometer $(V S M)$ was $\chi_{\text {PARA }}=4.4 \times 10^{-5} \mathrm{emu} / \mathrm{g}$.

The bulk crystal sample was ground to a micron size and placed in the ampoule. The ampoule was connected to the sample chamber by a copper pipe. The interior of the chamber, the ampoule and the pipes were filled with He gas at $1.0 \times 10^{5} \mathrm{~Pa}$. Thereafter, additional He gas was supplied to the ampoule at an enhanced pressure of about $1.1 \times 10^{5} \mathrm{~Pa}$ for about $1 \mathrm{~s}$, and the sample grains were dispersed into the He gas. The gas with dispersed grains was injected into the chamber. The degree of grain alignment at arbitrary field intensity is obtained from the intensity of the $\mathrm{He}$ Ne laser beam transmitted through the sample suspension in a direction parallel to the magnetic field. This can be done because the direction of an optical principle axis of a crystal grain coincides with the direction of the magnetic principle axis. The efficiency of the above method has been established in earlier experiments (Uyeda et al., 2001, 2003). A small portion of the dispersed grains was collected on a copper plate immediately after each experimental run was completed. $M$ values at the time of the experiment were estimated as $M=(1.9 \pm 1.0) \times 10^{-14} \mathrm{~g}$, which was obtained from the size distribution $(1.1 \pm 0.5 \mu \mathrm{m}$ in diameter) observed on scanning electron microscope (SEM) images and volume density $\rho=3.2 \mathrm{~g} / \mathrm{cm}^{3}$. 


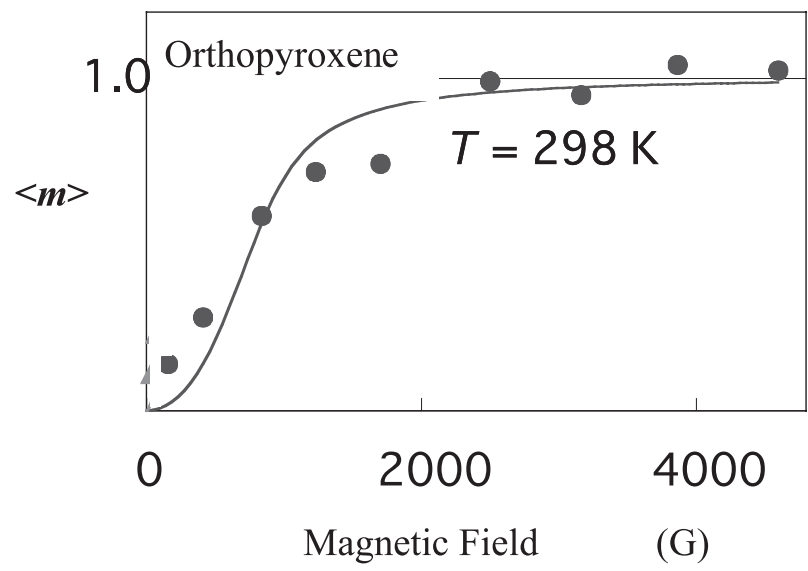

Fig. 2. Magnetic alignment of ortho-pyroxene grain observed at room temperature $(T=300 \mathrm{~K})$. Solid symbols indicate measured relationships; solid curve represents theoretical fit to the measured data. Degree of alignment is described by an order parameter $\langle m\rangle$; its definition is given in the text.

\section{Measured Results of Magnetic Grain Align- ment}

Results of the grain alignment are shown in Fig. 2 for ortho-pyroxene grains. The degree of the alignment is defined by an order parameter $\langle m\rangle$, which is an average of $1 / 2\left(3 \cos ^{2} \theta-1\right)$ over an ensemble of measured grains. Here $\theta$ denotes an angle between a magnetically stable axis of a grain and $B$. The average $\theta$ value at a completely random state is $54.7^{\circ}$, whereas $\theta$ is equal to $0^{\circ}$ at a completely ordered state. The completely ordered and random states are described as $\langle m\rangle=1$ and $\langle m\rangle=0$, respectively. The parameter $\langle m\rangle$ has been conventionally used to describe an aligned state of liquid crystal. An almost full alignment is achieved at a field below $2000 \mathrm{G}$. It has not been considered possible for magnetic grain alignment to occur at such a low field intensity for oxide particles without spontaneous magnetic moment; however, we have achieved alignment in the field of a simple hand magnet. A similar effect caused by paramagnetic ions was also observed for common silicates, such as biotite, muscovite, orthoclase, talc and kaolinite (Uyeda et al., 2003, 2005a).

A theoretical fit to measured data is calculated from a Boltzmann average of $1 / 2\left(3 \cos ^{2} \theta-1\right)$ in terms of a field-induced free energy of particle $U=$ - $\left(M B^{2} / 2\right)\left\{\chi_{\perp}+\Delta \chi \cos ^{2} \theta\right\}$; magnetic anisotropy $\Delta \chi$ is described as $\Delta \chi=\chi_{\|}-\chi_{\perp}$, where $\chi_{\|}$and $\chi_{\perp}$ are the susceptibilities parallel and perpendicular to the stable axis, respectively. Here, a $M \Delta \chi$ value which gives the best fit to the measured data is used to describe the curves. The adopted $M \Delta \chi$ value in the above calculation was $M \Delta \chi=(4.3 \pm 2.1) \times 10^{-19} \mathrm{emu}$, which is consistent with the value obtained from the experimental $M$ and $\Delta \chi$ values; $M \Delta \chi=(2.3 \pm 1.1) \times 10^{-19} \mathrm{emu}$. The consistency indicates that alignment proceeded by a Langevin process.

\section{Effect of $M, \Delta \chi$ and $T$ on the Alignment Process}

A field intensity where the anisotropy energy $(1 / 2) M \Delta \chi B^{2}$ of the particle was equal to $(15 / 2) k_{\mathrm{B}} T$ was defined as field of alignment $B_{\mathrm{S}}$ (Uyeda et al., 1993),

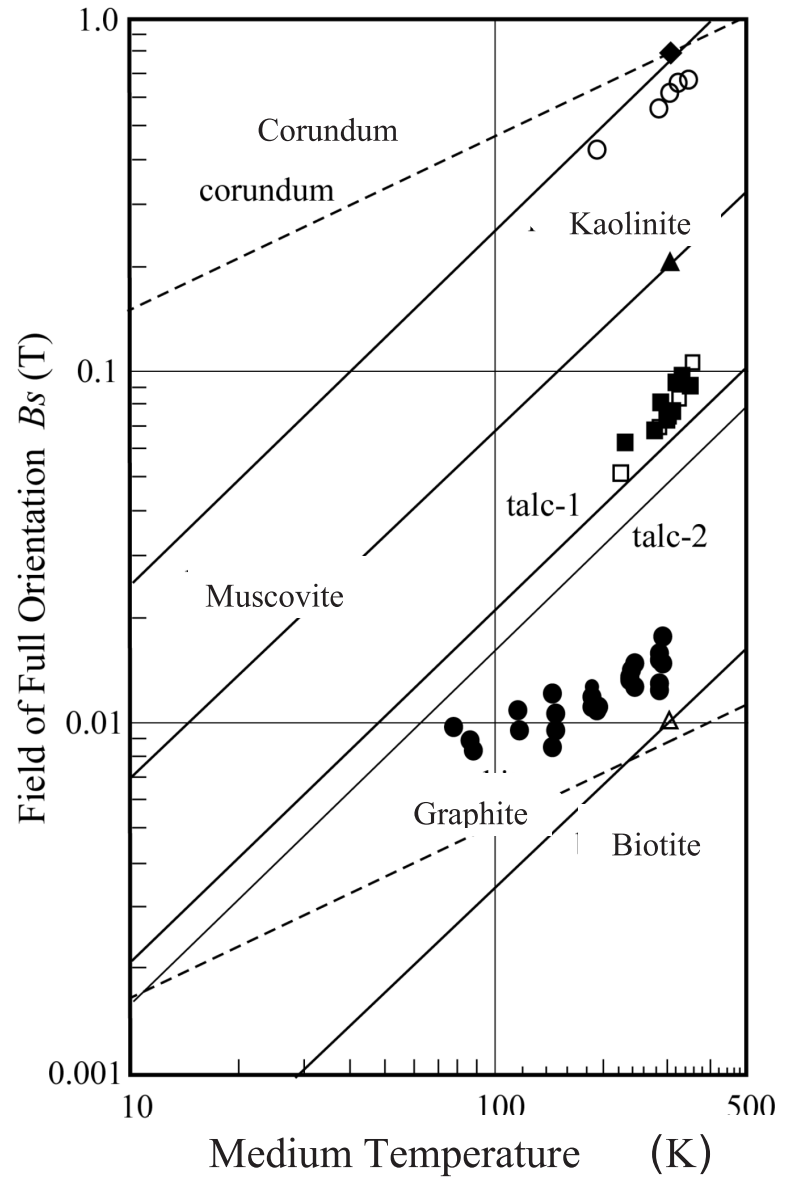

Fig. 3. Relationship between field of orientation $B_{\mathrm{s}}$ and temperature compiled for various silicate (Uyeda et al., 2005b). Definition of $B_{\mathrm{s}}$ is given in the text. Experimental data of graphite (solid circles) are measured by the system described in Fig. 1.

which was directly calculated from the Langevin process as

$$
B_{\mathrm{s}}=\left(15 k_{\mathrm{B}} T / N \Delta \chi\right)^{1 / 2} .
$$

The value of $\langle m\rangle$ is equal to 0.78 when the external field is equal to $B_{\mathrm{s}}$. The equation explicitly shows that the field required to align a particle will decrease as $T$ decreases and also decrease as either $M$ or $\Delta \chi$ increase. Complete alignment is achieved only at a infinite field intensity. Therefore, it is necessary to introduce an indicator, such as $B_{\mathrm{s}}$, in order to carry out quantitative analysis on the alignment process (Uyeda et al., 2005b). In a typical study of magnetic grain alignment, a theoretical $B_{\mathrm{s}}$ value is calculated by inserting observed $M, \Delta \chi$ and $T$ values in Eq. (1), and the obtained value is compared with an experimental $B_{\mathrm{s}}$ obtained from the measured $\langle m\rangle-B$ relationship. When the alignment proceeds by the Langevin process, the two independently obtained $B_{\mathrm{s}}$ values show fairly good agreement. The effect of parameters $M, \Delta \chi$ and $T$ on $B_{\mathrm{s}}$, which is expected from Eq. (1), has been systematically examined by measuring common sheet-silicates, such as muscovite, talc and kaolinite (Uyeda et al., 1991, 1993). The results of these experiments confirmed that the field of alignment could be reduced considerably by increasing $M$ or $\Delta \chi$, and also by decreasing $T$, as expected from Eq. (1) (Uyeda $e t$ al., 2005a). 
The effect of $M$ on the $B_{\mathrm{s}}$ value was pointed out by Yamagishi et al. (1989) in the course of their analysis of the polymerization process of fiblin fibers in a magnetic field. The effect of $M$ was also discussed by Sazaki et al. (1989) when they analyzed the field dependence of the growth of lythozyme crystals. However, these latter researchers found that the reduction rate of $B_{\mathrm{s}}$ did not exceed $10 \%$ due to increased $M$, since the maximum size of the particle that could not be maintained in the dispersed fluid did not exceed several microns in terrestrial gravity. Chihara et al. (1998) measured the temperature dependence of $B_{\mathrm{s}}$ for graphite grains free of paramagnetic ions in a temperature range of $T=300 \mathrm{~K}$ and $180 \mathrm{~K}$, as shown in Fig. 3. The grains were dispersed in liquid ethanol. A $T^{1 / 2}$ dependence, expected from Eq. (1), was confirmed in this experiment.

For the effect of $\Delta \chi$, paramagnetic anisotropy $\Delta \chi_{\text {PARA }}$ is a dominant factor compared to diamagnetic anisotropy $\Delta \chi_{\text {DIA }}$ for most of the natural oxides. The origin of $\Delta \chi_{\text {DIA }}$ was attributed to preferential orientation of bond direction with respect to a magnetic principle axis of crystal (Uyeda, 2004a). Accordingly, the $\Delta \chi_{\text {DIA }}$ of various silicates was expected to distribute in a range between $10^{-8}$ and $10^{-10} \mathrm{emu} / \mathrm{g}$. In contrast, $\Delta \chi_{\text {PARA }}$ is derived from the anisotropy of the local crystalline field of a site that is occupied by an isolated paramagnetic ion (Ballet and Coey, 1982). Hence, $\Delta \chi_{\text {PARA }}$ is proportional to the concentration of the paramagnetic ion; it reaches a level of $10^{-5} \mathrm{emu} / \mathrm{g}$ when the concentration of $\mathrm{Fe}^{2+}$ is about $1 \mathrm{~mol} \%$. Such concentrations of $\mathrm{Fe}^{2+}$ are often observed in major rockforming minerals. The effect of paramagnetic $\mathrm{Fe}^{2+}$ ions in reducing in $B_{\mathrm{s}}$ at room temperature was specifically observed for biotite grains, which is a typical sheet-silicate (Uyeda et al., 2005a). The value of $B_{\mathrm{s}}$ was found to be as low as 220 Oe for biotite, which had a susceptibility of $\chi_{\text {PARA }}=4.4 \times 10^{-5} \mathrm{emu} / \mathrm{g}$. This $B_{\mathrm{s}}$ was less than $1 \%$ of the $B_{\mathrm{s}}$ value observed for synthetic phlogopite that was free of paramagnetic ions, whereas the $B_{\mathrm{s}}$ was $6.2 \mathrm{kOe}$ for phlogopite with $\chi_{\text {PARA }}=1.2 \times 10^{-6} \mathrm{emu} / \mathrm{g}$. The concentration of $\mathrm{Fe}^{+2}$ is proportional to $\chi_{\text {PARA }}$ in the above three minerals. As almost equivalent crystal structures are reported for these minerals, they should have similar amounts of $(\Delta \chi)_{\text {DIA }}$. Hence, the large variation of $B_{\mathrm{s}}$ observed between the three materials can be attributed to the difference in the paramagnetic $(\Delta \chi)_{\text {PARA }}$.

Variations in the temperature are expected to change the values of $(\Delta \chi)_{\text {PARA }}$ and $B_{\mathrm{S}}$ considerably since the paramagnetic moment follows a Cuire-Weiss law. The $\Delta \chi-T$ relationships were previously measured for bulk single crystals at between $T=300 \mathrm{~K}$ and $100 \mathrm{~K}$ for biotite and muscovite that contained $\mathrm{Fe}^{2+}$ ion at a level of $1 \mathrm{~mol} \%$. As temperature decreased from $T=300 \mathrm{~K}$ to $80 \mathrm{~K}$, the $\Delta \chi$ of biotite increased from $1.2 \times 10^{-5} \mathrm{emu} / \mathrm{g}$ to $8.0 \times 10^{-5} \mathrm{emu} / \mathrm{g}$, while the $\Delta \chi$ of muscovite increased from $0.9 \times 10^{-6} \mathrm{emu} / \mathrm{g}$ to $5.1 \times 10^{-6} \mathrm{emu} / \mathrm{g}$ (Uyeda et al., 2004b). The temperature dependence of $B_{\mathrm{s}}$ was measured between $T=300 \mathrm{~K}$ and $200 \mathrm{~K}$ for crystal grains of talc and kaolinite that contained paramagnetic ions (Uyeda et al., 2003). As shown in Fig. 3, observed $B_{\mathrm{s}}$ of kaolinite and talc was nearly proportional to $T$ since $(\Delta \chi)_{\text {PARA }}$ is proportional to $T^{-1}$ following Curie's law. Here, the magnitude of $(\Delta \chi)_{\text {DIA }}$ was negligible compared to $(\Delta \chi)_{\text {PARA }}$. Hence, when a material contains a finite amount of paramagnetic ion, the reduction rate of $B_{\mathrm{s}}$ with decreasing $T$ becomes much larger than the above-mentioned $T^{1 / 2}$ dependence of a diamagnetic material. The measurement below $T=273 \mathrm{~K}$ involves using liquid ethanol as the dispersing medium; at the present time, pure water is used as the dispersing medium in most measurements.

In conclusion, most of the diamagnetic oxides have a potential to cause magnetic alignment, with the exception of cubic crystals and amorphous materials. The underlying reason for this is that the resultant $\Delta \chi$ may be enhanced considerably by increases in the concentration of paramagnetic ions. $\Delta \chi$ increases even more with decreasing temperature.

\section{Conditions Required to Reproduce Dust Align- ment in Planetary Formation Region}

The values of $\chi_{\text {PARA }},(\Delta \chi)_{\text {PARA }}$ and $B_{\mathrm{s}}$ observed for ortho-pyroxene are comparable to those of the biotite mentioned above. Consequently, $B_{\mathrm{s}}$ is expected to be considerably reduced in the low-temperature region below $T=$ $100 \mathrm{~K}$, which is now in progress for ortho-pyroxene. Precise measurements of both $\Delta \chi$ and $B_{\mathrm{s}}$ are required in the low-temperature region for ortho-pyroxene as well as for other dust minerals. These results may provide quantitative information that can be used to assess whether dust alignment in planetary formation region is caused by a simple Langevin process or not.

The magnetic field is known to be a major factor that controls the evolution of stars and planets. For example, reconnection of magnetic field associated with the protoplanetary disk was recently proposed to be a heat source that produces high-temperature condensates of primitive meteorites (Shu et al., 1997). In order to assess this hypothesis on planetary evolution, it is necessary to carry out high-spatial-resolution polarimetery mapping (about $1 \mathrm{AU}$ ). Near infrared (Lucas et al., 2004) and sub-millimeter (Tamura et al., 1999) imaging polarimetry was recently reported for YSO regions as well as for massive young objects (Momose et al., 2001). Spatial resolutions in these observations were about $100 \mathrm{AU}$ although the data indicate that partial magnetic alignment of dust particles does take place in these systems. It is expected that polarimetry surveys with high-spatial resolution will be realized by forthcoming projects such as that of the Akatama Long Mand Association (ALMA).

It is necessary to reproduce the temperature conditions of planetary formation regions, which range between $400 \mathrm{~K}$ and $50 \mathrm{~K}$; the setup shown in Fig. 1 is a step towards realizing alignment in a temperature range between $T=200 \mathrm{~K}$ and $50 \mathrm{~K}$. In addition, our apparatus is capable of performing magnetic alignment in a reduced temperature condition down to $T=10 \mathrm{~K}$, which is comparable to that of the molecular cloud. The experiment was possible because He gas is a unique medium that can disperse micron-sized particles and remain in the liquid state above $T=4.2 \mathrm{~K}$. At the present time, measurements are only possible at temperatures above $160 \mathrm{~K}$ for silicate grains since liquid ethanol is used as a dispersing medium. As mentioned before, it is 
essential to know the geometrical relationships between $B$ and $P$ for determining the field direction from polarimetry data. This relationship differs considerably depending on the alignment model used. The apparatus shown in Fig. 1 is capable of examining the relationship between $P$ and the direction of the crystalline axes of mineral grains, which is not clearly understood in the dense region.

Acknowledgments. The authors wish to thank Dr. Anne Hofmeister for valuable comments and discussions.

\section{References}

Ballet, O. and J. M. D. Coey, Magnetic-properties of sheet silicates-2-1 layer minerals, Phys. Chem. Miner., 8, 218-229, 1982.

Chihara, H., C. Uyeda, A. Tsuchiyama, and T. Yamanaka, The magnetic ordering of graphite grains and an experimental application to grain alignment, Astron. Soc. Jpn., 50,149-154, 1988.

Hanner, M. S., T. Y. Brookie, and A. T. Tokunaga, 10 micron spectroscopy of young stars in the Rho Ophiuchi cloud, Astrophys. J., 438, 250-258, 1995.

Hofmeister, A. M., B. Wopenka, A. J. Locock et al., Spectroscopy and structure of hibonite, grossite, and CaAl2O4: Implications for astronomical environments, Geochem. Cosmochem. Acta, 68, 4485-4503, 2003.

Honda, M., H. Kataza, Y. K. Okamoto, T. Miyata, T. Yamashita, S. Sako, S. Takubo, and T. Onaka, Detection of crystalline silicates around the T Tauri star Hen 3-600A, Astrophys. J., 585, L59-L63, 2003.

Honda, M., H. Kataza, Y. K. Okamoto, T. Miyata, T. Yamashita, S. Sako, T. Fujiyoshi, M. Ito, Y. Okada, I. Sakon, and T. Onaka, Crystalline silicate feature of the Vega-like star HD 145263, Astrophys. J., 610, L49-L52, 2004.

Langevin, P. and P. Curie, Magnetisme ettheorie des electrons, CP Acad. Sci. Paris, 151, 331-368, 1910.

Lucas, P. W., M. Fukagawa, M. Tamura, and A. Beckford, High-resolution imaging polarimetry of HL Tau and magnetic field structure, Mon. Notic. Roy. Astron. Soc., 352, 1347-1364, 2004.

Malfait, K., C. Waelkens, L. B. F. M. Waters, B. Vandenbussche, E. Huygen, and M. S. de Graauw, The spectrum of the young star HD 100546 observed with the Infrared Space Observatory, Astron. Astrophys., 332, L25-L28, 1998.

Maret, G. and K. Dransfield, Biomolecules and polymers in high steady magnetic filed, Topics Appl. Phys., 57, 144-204, 1985.

Molster, F. J., L. B. F. M. Walters, A. G. G. M. Tielens, C. Koike, and H. Chihara, Crystalline silicate dust around evolved stars. III. A correlations study of crystalline silicate features, Astron. Astrophys., 382, 241-255, 2002.

Momose, M., M. Tamura, O. Kameya, J. S. Greaves, A. Chrysostomou, J. H. Hough, and J. I. Morino, Submillimeter imaging polarimetry of the NGC 7538 region, Astrophys. J., 555, 855-862, 2001.
Shu, H. F., H. Shang, A. E. Glassgold, and T. Lee, X-rays and fluctuating X-winds from proto-stars, Science, 277, 1475-1497, 1997.

Sitko, M. L., C. A. Grady, D. K. Lynch, R. W. Russel, and M. S. Hanner, Cometary dust debris disks of HD 31648 and HD 163296, Astrophys. J., 510, 408-412, 1999.

Spizer, L., Jr., Physical Properties in the Interstellar Medium, Wiley \& Sons Inc., New York, 1978.

Tamura, M., J. H. Hough, J. S. Greaves, J. I. Morino, A. Chrysostomou, W. S. Holland, and M. Momose, First detection of submillimeter polarization from T Tauri stars, Astrophys. J., 525, 832-836, 1999.

Uyeda, C., T. Takeuchi, A. Yamagishi, and M. Date, Diamagnetic orientation of clay mineral grains, J. Phys. Soc. Jpn., 60, 3234-3237, 1991.

Uyeda, C., T. Takeuchi, A. Yamagishi, T. Yamanaka, A. Tsuchiyama, and M. Date, Diamagnetic anisotropy of sheetsilicates, Phys. Chem. Miner., 20, 369-374, 1993.

Uyeda, C., M. Sakakibara, and H. Chihara, Magneto-rotation experiment of diamagnetic single-crystals suspended in the gas medium for examining dust alignment in interstellar region, J. Phys. Soc. Jpn., 70, 12261229, 2001.

Uyeda, C., M. Sakakibara, and K. Tanaka, Temperature dependence observed of magnetic rotation process of micron sized kaolinite, Phys. Chem. Miner., 30, 425-429, 2003.

Uyeda, C., R. Takashima, and K. Tanaka, Mangeto-rotation of nonmagnetic micro-crystals caused by diamagnetic anisotropy, Appl. Phys. Lett., 28, 094103, 2004a.

Uyeda, C., K. Tanaka, R. Takashima, and M. Sakakibara, Magnetic alignment at reduced field intensity due to decrease of temperature observed for nonferromagnetic particles possessing paramagnetic and diamagnetic anisotropy, Jpn. J. Appl. Phys., 43, 980-984, 2004b.

Uyeda, C., in Magneto-Science, edited by Tanimoto et al., pp. 324, Kodansha Co. Tokyo, 2004c.

Uyeda, C., R. Takashima, and K. Hiraoka, Magnetic alignment of nonmagnetic mica crystals achieved at low field intensity due to high concentration of paramagnetic ions, Jpn. J. Appl. Phys., 44, L371-L374, $2005 a$.

Uyeda, C., M. Sakakibara, K. Tanaka, and R. Takashima, Experiment of fine graphite-crystals dispersed in He gas oriented to study alignment of crystalline-axes of nano-sized non-magnetic particles, J. Electron. Microsc., 43, 1-5, 2005 b.

Whittet, D. C. B., Dust in the Galactic Environment, p. 110, Institute of Physics Publishing Bristol, 1992.

Willacy, K. and W. D. Langer, The importance of photoprocessing in protoplanetary disks, Astrophys. J., 544, 903-920, 2000.

Yamagishi, A., T. Takeuchi, H. Higashi, and M. Date, Diamagnetic orientation of polymerized molecules under high magnetic-field, J. Phys. Soc. Jpn., 58, 2280-2283, 1989.

C. Uyeda (e-mail: uyeda@ess.sci.osaka-u.ac.jp), K. Hisayoshi, and S. 